\title{
A State-of-the-Art Study on Energy Harvesting Systems: Models and Issues
}

\author{
Rahul Yadav ${ }^{1}$, Ayush Goel' ${ }^{1}$, Shruti Vashist ${ }^{1}$ and Mohit Verma ${ }^{1,2, *}$ \\ ${ }^{1}$ Department of Electronics \& Communication Engineering, Manav Rachna University, Faridabad 121001, India; \\ rahulrkrules25@gmail.com, ayushoct29@gmail.com, shruti.fet@mriu.edu.in \\ 2Accendere CL Educate, New Delhi 110044, India; mohit.verma@accendere.co.in
}

\begin{abstract}
Background/objectives: Energy is highly essential for the life of living beings. As technologies are getting advanced, the consumption of energy increases continuously. Conventional sources available at earth are limited, and it will be going to drain day by day. Therefore, it is necessary to design models which are capable of harvesting energy using natural resources. The main objective of this paper is to summarise recent contributions in the area of energy harvesting (EH) and discuss their models with operating process, advantages, and limitations. Also these models are compared among themselves in terms of energy generation capacity. Methods/statistical analysis: Several models have been developed for $\mathrm{EH}$ by researchers all over the world. Here, an attempt is made to review the various models involved in EH to prevent the deficiency of energy. Findings: An EH technique is one of the most potential methods to encounter the energy deficiency problem. This study describes few models dedicated to EH and focuses on the major issues such as the necessity of highly efficient electronic circuits for capturing, accumulating, and storing even small electrical energy. Also the harvester circuit must stay in the active mode and be ready to perform energy capturing whenever harvestable energy becomes available. Nowadays, several sources (non-conservative) are used for EH such as warmth of human body. In future, the main focus is to enhance the efficiency of the energy harvester system. Improvements/application: Energy consumption is increasing day by day and its shortage is already predicted in the near future. Therefore, techniques for the generation of uninterrupted power provide for the incessant operation of any device to make life easier.
\end{abstract}

Keywords: Energy Harvesting, Thermo-generator, Classical Energy Harvesting Model, Generic Sensor Network Node, Energy Harvesting Using Super Capacitor

\section{Introduction}

A $5 \mathrm{G}$ network design is significantly encouraged by energy-efficient characteristics. Huge attention has been paid to the hikes in the cost of electricity for network operations besides its adverse effects on the environment.

In a mobile network, $80 \%$ of the total power consumption is attributed to the base station. ${ }^{1}$ At present, the available theory highlights the recent contributions in energy efficiency and other network performance indicators such as delay, bandwidth, etc., of the system. One of the potential solutions to combat the challenges/ problems related to energy inefficiency is energy harvesting
(EH). In this process, the major focus is to produce energy from environmental-friendly sources such as sun (solar cell) and wind turbine. The main advantages of these sources are their capabilities to generate energy without $\mathrm{CO}_{2}$ emissions, which prevents adverse effects on the environment. Solar solutions suit most of the countries in Asia and Africa due to the immense availability of sunlight at day time. On the other hand, wind turbine solutions are more appropriate for central European and Scandinavian countries due to cloudy and windy weather.

For implementing $\mathrm{EH}$, few challenges need to be taken care of by the network designer. In a systematic power supply resolution, a constant quantity of power is

*Author for correspondence 
available throughout the operation, whereas $\mathrm{EH}$ solutions are time-dependent and the availability of energy is a stochastic process, $\underline{2}$ for example, sunlight is not available at night. Therefore, the harvesting period is idle for a long time. Here various EH models are discussed with their operating processes, advantages, and limitations. Also, these models are compared among themselves in terms of their energy generation capacity.

\section{Study of Different Types of Models}

\subsection{EH Using Thermo-generator}

A thermogenerator ${ }^{3}$ is a device which generates an electrical charge from the heat of the human body (mostly from wrist). It is based on the see beck effect, $\underline{\underline{4}}$ which is a phenomenon in which a temperature difference between two different electrical conductors or semiconductors produces a voltage between two substances. The generated voltage is directly proportional to the temperature difference between the two platforms known as cold junction and hot junction.

The entry and exit of energy at the junction is totally based on two facts:

1) The available temperature gradient at junctions.

2) Absorption or dismissal due to Peltier effect $\underline{\underline{5}}$ (which is defined as a temperature difference between two electrodes connected to a semiconducting material arising due to an applied voltage). This phenomenon is useful when one has to transfer heat from one medium to another on a small scale.

In a thermoelectric module, $\underline{6}$ the generator consists of a thermocouple, which includes both types of semiconductors: $\mathrm{p}$-type and n-type, having connection in series-wise electrically and parallel-wise thermally. Due to the see back effect, voltages arise, thus there is different temperature at the thermocouple ends. The connection of electrical circuit is made in such a manner that it allows adding the voltages obtained at each thermocouple and finally the total output voltage arises at the thermoelectric generator (TEG) end. This obtained output voltage is directly proportional to the number of thermocouples present and to the temperature gradient between the cold and the hot side.

In 2007, Seiko Thermic reached a significant milestone. ${ }^{7}$ They precisely used a TEG to convert heat from human wrist into electric energy. It was the first watch to operate by taking the power by the temperature gradient between the body and environment. TEG produces a power of at least $1.5 \mu \mathrm{W}$ when the temperature difference was in between the range from $-3^{\circ} \mathrm{C}$ to $1^{\circ} \mathrm{C}$ (here the temperature range indicated the heat absorbing range of watch from the wrist). A demo model of TEG is shown in Figure 1, which converts body heat into some amount of energy (nearly $1.5 \mathrm{~V}$ ) to operate a watch and some specific embedded medical devices used to measure or monitor blood pressure, heart rate, etc. With a temperature difference of $5^{\circ} \mathrm{C}$ and a surface area of 0.5 $\mathrm{cm}^{2}$, TEG generates approximately $40 \mu \mathrm{W}$ of power at $3 \mathrm{~V}$. This generated electricity is stored in a thin-film lithium battery.

A compact TEG model ${ }^{\underline{8}}$ was established whose output is in equilibrium with the macroelectronic system load. The working limit of a TEG is at $273 \mathrm{~K}$ which is the room temperature, but its limits do not extend beyond $120^{\circ} \mathrm{C}$, due to which it provides the output power of $20 \mu \mathrm{W}$.

\subsubsection{Design Considerations}

The human body is a great source of energy which may act as the temperature difference between the bodies and the environment, which is accepted by the TEG to get electrical energy. In 1994, Stunner- concluded that the efficiency between temperature ranges of $20-35^{\circ} \mathrm{C}$ is $5.5 \%$. In a warmer region, the efficiency drops compared to a colder region due to a rise in heat energy.

The placement of device in the body part is very crucial. It is suggested that the neck area would be the best to place this device as it is one of the warmest body parts and also easily reachable. It was observed that the generated power using TEG lay within the range of $0.2-0.32 \mathrm{~W}$. The

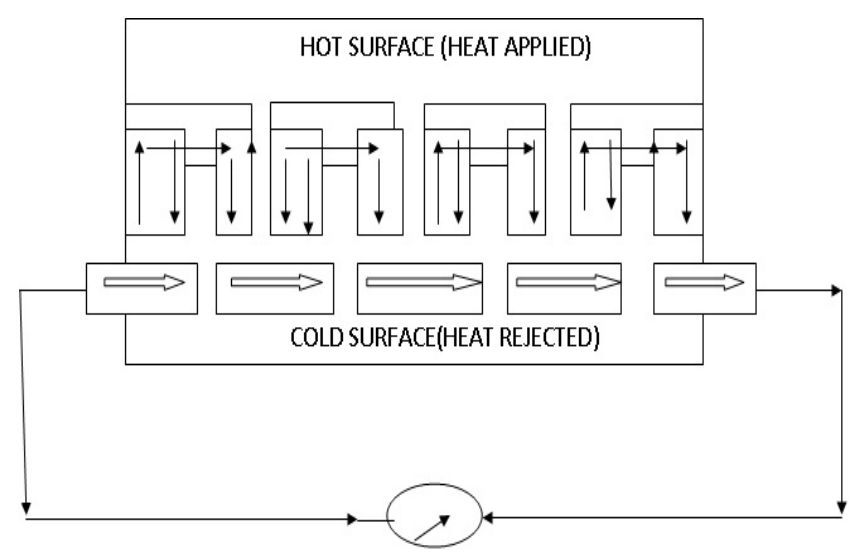

Figure 1. A thermoelectric generator. 
material used for fabricating this device also plays a vital role. Aaltenkirch 10 suggested that a good thermoelectric material should have large see beck coefficients and large electrical conductivity with low thermal conductivity. These parameters are easily related with the figure of merit, given by

$$
Z=\frac{\alpha * 2 * \sigma}{\lambda}
$$

where $\alpha$ is see beck coefficient; $\sigma$ is electrical conductivity; $\lambda$ is thermal conductivity; and $Z$ is figure of merit.

The design of a TEG also requires proper dimensions. Again, the maximum obtained driving power by TEG is inversely proportional to the area of cross-section (A) and directly proportional to the length of $\mathrm{p}-\mathrm{n}$ thermoelectric leg couple. This leg length is also inversely proportional with power density $(D)$. Again, specific power $\left(\mathrm{W} / \mathrm{cm}^{2}\right)$ will increase if the height of thermoelectric elements reduces, while maintaining the same aspect ratio of their legs. In 2006, 11 it was theoretically concluded that specific power can be produced at room temperature if the value of figure of merit is equal to 0.9 at $10 \mathrm{~K}$. The efficiency achieved by a thermoelectric microconverter is approximately only $5-6 \%$. Ryan et al. depicted that conversion efficiency can be improved by manipulating the electrical and thermal transports on nanoscale; this will also enhance the figure of merit by a factor of $2.5-3 \%$ near room temperature.

\subsubsection{Power Management Unit}

It is necessary to design the power module in such a way that it converts the input voltage lying within few hundreds of $\mathrm{mV}$ into a higher output voltage $(\mathrm{V})$. It is also necessary to have at least $0.8 \mathrm{~V}$ for starting to regulate a conventional step-up converter. Again, the required specified energy transfer could not be performed by a single converter module. Therefore, it is highly necessary to design an optimum power management system for uninterrupted operations. The designed power management contained a charge pump in conjunction $\underline{12}$ with a step-up DC to DC converter. $\underline{13}$ This charge pump is responsible for delivering the necessary start-up voltage to the switching circuitry of the step-up converter, which undertakes energy conversion once activated.

The power consumption of a thermogenerator is too high; therefore it is very difficult to implement it on a continuous RX path (receiving end path). Along with the lack of implementation of permanent receiver capabilities, the entire system will have a defect as the amount of energy harvested cannot be collected.

\subsection{Classic EH Model}

Figure 2 represents a block diagram of a classic EH. This block consists mainly of five different blocks on which the model is working. Below the working of different blocks is described briefly.

\subsubsection{Working of Different Blocks}

Energy generator: The energy generator is fabricated using a piezoelectric fibre composite which generates the piezoelectric effect (it is defined as the ability of a certain material to generate an electric charge in response to an applied mechanical stress).

Detector: The function of the detector is to detect, calculate, and estimate the amount of power received into the EH system in order to drive any desired application. The detector also has an in-built sensor, which alerts the system about the power available or power consumed by the load.

EH module/energy storage: The function of $\mathrm{EH}$ module/ energy storage is to properly manage the power received from the system. It also manages power distribution from the system.

Switch: The switch allows and restricts the passage of harvested energy to the load.

Load: The load is finally the power consumer part of the system. There are various sources such as microprocessor sensors, wireless sensor network transceivers, etc., using the harvested energy as their energy source.

\subsubsection{Working Procedure}

As soon as the energy is generated from the transducer, the detector electronics receives the injected energy from the energy generating source. This electrical energy is now accumulated, collected, and stored in the internal storage unit such as a battery, capacitor, and supercapacitor. Energy is captured by operating it between two supply

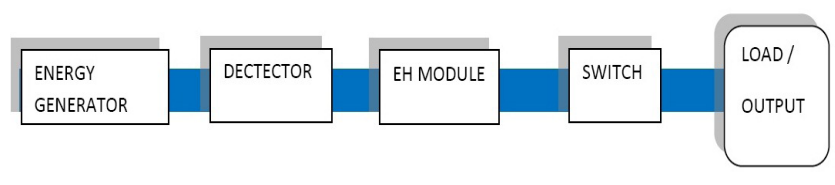

Figure 2. Block diagram of a classic EH model. 
threshold voltages, i.e. $\mathrm{V}_{\min }$ at low $\mathrm{DC}$ and $\mathrm{V}_{\max }$ at high DC; these maximum and minimum voltages are set in accordance with the requirement of load applications.

Once the maximum voltage is received, the system terminates further charging and sets the output on "on demand requirement" to power the load. As the output power goes down and reaches its minimum, the charging cycle begins again and attains the maximum value. This process is continuously running by taking nearly $10 \mathrm{~mA}$ current as input. The same process charge/cycle times repeat every 4 minutes at an average input current of 10 $\mathrm{mA}$. To obtain optimum performance and long energy retention time, $\mathrm{EH}$ electronics need to be so designed such that it consumes energy much smaller than the energy input by the generating source, which leads the design to incorporate micropower devices.

\subsection{Electric Generic Sensor Node $\underline{\underline{14}}$}

An electric generic sensor node harvests electric energy from a mechanical source. In this proposed technique, mechanical stress is used for EH. The system uses a piezoelectric transducer, such as a Teminc piezo atomiser, microporous atomiser or metal mesh atomiser, etc., to harvest energy. It has been proposed to be implemented mainly on an overbridge or flyover. The transducer system is physically placed over the surface of the bridge/ flyover. Whenever a vehicle passes through the bridge or flyover, the pressure applied to the transducer converts this mechanical energy into electrical energy. These transducers are connected to the sensor node which accumulates all the energy generated by the transducer and sends to the powerhouse. $\frac{15}{}$ These fabricated systems are sensible/capable enough to detect a minor human stepping and convert it into power. The block diagram of an electric generic sensor node is shown in Figure 3.

The different parts of the proposed model are $\frac{16}{6}$ :

Sensing plates: These are inscribed over the surface of a flyover to detect the movement of mechanical pressure on the overbridge. The system is designed in such a way that whenever there is a passage of vehicle over the sensing plates, the system will generate electricity from the load applied to the plates.

Counter: The main function of a counter is to count the amount of movements over the bridge and send it to the transducer.

Transducer: The main function of a transducer is to convert one form of energy into another. In energy

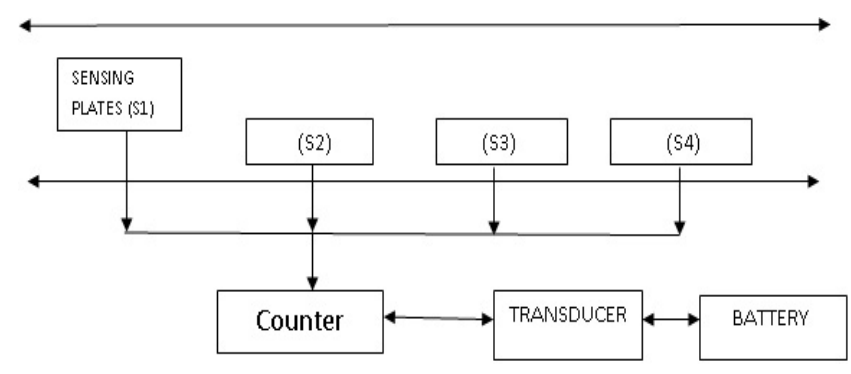

Figure 3. Block diagram of an electric generic sensor node.

generic sensor node, a piezoelectric traducer is used. As implied by the name, the transducer is based on piezoelectric effect and converts mechanical energy into electric energy.

Battery: The battery is used for the storage of harvested energy.

\subsection{EH Using Supercapacitor}

EH based on a supercapacitor uses three sources of power at the same time. These sources are solar panel, battery and supercapacitor. These power sources switch among themselves as per the requirement of the system. A key component of the system is a microcontroller, which is used to collect the data and take a decision regarding switching the source according to the requirement. The transducer is used to monitor the output sources. If solar energy is not available due to climatic conditions, the reserve source, i.e. battery, will be utilised. At first, the transducer observes weather conditions on the basis of the intensity of sunlight, then it sends the observed data to the microcontroller to take a decision on switching. ${ }^{17}$ Figure 4 represents the block diagram of the system. The different components of the system are described below:

Sensor: The sensor used in the supercapacitor is temperature sensor. It is a linear-type semiconductor $\frac{13}{3}$ Lm35 or Lm32, which can produce an output of magnitude in the range of $\mathrm{mV}$. This produced output voltage is proportional to the temperature. The working temperature range of these transducers is $+/-1 / 4^{\circ} \mathrm{C}$ at room temperature and $3 / 4^{\circ} \mathrm{C}$ in overall range of $-55^{\circ} \mathrm{C}$ to $+150^{\circ} \mathrm{C}$ without any external calibration or trimming.

Solar panel: The solar panels are composed of monocrystalline silicon. These solar panels are arranged in the wafer form having a thickness in-between 160 and $240 \mathrm{~mm}$. These panels are small and provide better output, which helps to reduce the need for MPPT (maximum power point tracking) technique. 


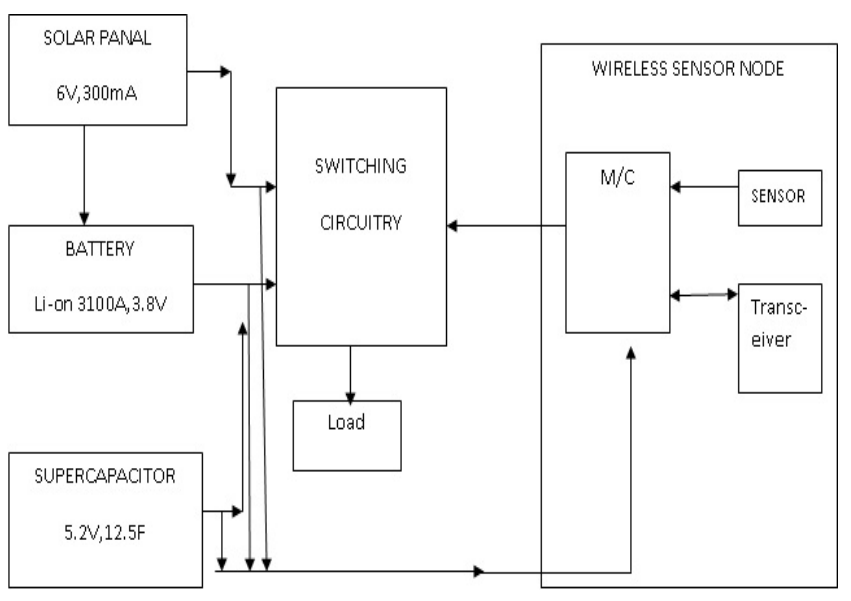

Figure 4. Energy harvesting using supercapacitor.

Supercapacitor: The supercapacitor used in this model is LSUC 25F, 2.6 V. The major reasons for selecting this capacitor are:

1) Availability of sufficient charging current for the capacitor

2) Time required for charging

3) Useable energy available for the capacitor.

The maximum charging current available from solar panels is $300 \mathrm{~mA}$. However, it is not possible to get a continuous output supply of $300 \mathrm{~mA}$. The capacitors are charged by current varying within the range of $0-300$ $\mathrm{mA}$. Another very important factor of charging is time. Solar EH has a limited time window during which the capacitor needs to be charged. The charging time of the capacitor depends upon the time constant and the maximum current available from the power source. These limitations necessitate the use of supercapacitors over normal capacitors.

Battery: A Li-ion battery of $3100 \mathrm{mAh}$ at $3.8 \mathrm{~V}$ is used for this system. Such a battery was selected due to the large number of recharge cycles, high charge density, low leakage, lack of memory effect, and sufficient voltage with one battery.

Wireless transceiver: A tanangf4 wireless transceiver was used to implement this $\mathrm{EH}$ model. The operating frequency of the transceiver is $2.4 \mathrm{GHz}$ which lies within the ISM band. The working principle of this wireless transceiver is the same as zigbee, which is specially built to control the sensor network of IEEE 802.15.4 standard in wireless personal area networks. This transceiver needs 3.3-3.6 V supply voltage and $45 \mathrm{~mA}$ of current during transmitting and $50 \mathrm{~mA}$ during receiving.
Microcontroller: The microcontroller is a key component of this model, which performs various functions such as monitoring the output of the system, collecting and accumulating the harvested energy and the task of decision making for the selection of the particular energy source according to the situation. The microcontroller used to implement this model was PIC18F4520.

The entire system works with the help of solar power, two supercapacitors (each of $25 \mathrm{~F}$ ), and the battery unit. $\underline{18}$ When the solar panel powers up the circuit, the battery and supercapacitor gets charged. The two capacitors are connected in series-wise which raises the voltage up to 5.2 $\mathrm{V}$ across them. The minimum required operating voltage of the system is $3.3 \mathrm{~V}$. This model was mainly designed to use less battery source, but the nodes need battery power to start the operation.

At first, the system reads the data received from sensors and collects the voltage from sources. The microcontroller then disconnects the battery and switches the solar panel mode if the voltage of the capacitor $\left(\mathrm{V}_{\text {cap }}\right)$ and solar panel $\left(\mathrm{V}_{\text {sol }}\right)$ is at their maximum value. The system will switch to the supercapacitor if $\mathrm{V}_{\text {cap }}$ is more than operating voltage, i.e. $3.3 \mathrm{~V}$. If the intensity of sunlight is good, this model is able to charge the supercapacitor and power the node simultaneously. Once the supercapacitor is fully charged, the processor will wait until the solar panel output falls below the threshold and then switches to the supercapacitor.

\section{Conclusion}

Various models have been developed to harvest energy and store it. This study described few models dedicated to $\mathrm{EH}$ and focused on the major issues such as the necessity of highly efficient electronic circuit for capturing, accumulating and storing even small electrical energy. Also, the harvester circuit must stay in the active mode and be ready to perform energy capturing whenever harvestable energy becomes available. Table 1 compares the various models described in this study.

In the future, these models will be embedded into a mobile handset to increase the potential of mobile battery. In the thermogenerator model, the warmth of human body is used to produce energy, whereas a piezoelectric transducer has been used in a classical energy model. However, the supercapacitor model employs three sources 
Table 1. Comparison of different energy sources

\begin{tabular}{|l|l|l|}
\hline Sl. no. & Energy source & Power density \\
\hline 1 & Solar energy (direct sunlight) & $100 \mathrm{mw} / \mathrm{cm}^{3}$ \\
\hline 2 & $\begin{array}{l}\text { Piezoelectric transducer (push } \\
\text { mechanism) }\end{array}$ & $50 \mu \mathrm{J} / \mathrm{N}$ \\
\hline 3 & Thermal (heat sink source) & $0.5-10 \mathrm{mw}$ \\
\hline
\end{tabular}

of power, i.e., battery, solar panel and supercapacitor, and source switching is carried out according to the situation. The microcontroller monitoring the output of the system collects and accumulates the harvested energy. The task of decision making for the selection of the particular energy source is based on a dynamic situation. In the energy generic sensor node, mechanical stress is converted into power with the help of a pressure transducer.

\section{Acknowledgement}

We would like to express gratitude to the Research Mentors of Accendere Knowledge Management Services Pvt. Ltd. for their comments on an earlier version of the manuscript. Errors are our own and shall not affect the reputations of these esteemed persons.

\section{References}

1. Ayang A, Ngohe-Ekam PS, Videme B, Temga J. Power consumption: base stations of telecommunication in sahel zone of cameroon: typology based on the power consumption-model and energy savings. J Energy. 2016;2016:1-15.

2. Ulukus S, Yener A, Erkip E, Simeone O, Zorzi M, Grover $\mathrm{P}$, et al. Energy harvesting wireless communications: a review of recent advances. IEEE J Sel Area Comm. 2015;33(3):360-81.

3. Lossec M, Multon B, Ahmed HB, Goupil C. Thermoelectric generator placed on the human body: system modeling and energy conversion improvements. Eur Phys J Appl Phys. 2010;52(1).

4. Van Herwaarden AW, Sarro PM. Thermal sensors based on the See beck effect. Sens Actuators. 1986;10(3-4):321-46.
5. Angrist SW. Direct energy conversion; 1933.

6. Ban S, Zhang J, Zhang L, Tsay K, Song D, Zou X. Charging and discharging electrochemical supercapacitors in the presence of both parallel leakage process and electrochemical decomposition of solvent. Electrochim Acta. 2013;90:542-9.

7. Kawata M, Takakura A, inventors; Seiko Instruments Inc, assignee. Thermoelectrically powered wrist watch. United States patent US 5,889,735. 1999.

8. Stordeur M, Stark I. Low power thermoelectric generatorself-sufficient energy supply for micro systems. In: XVI ICT'97. Proceedings ICT'97. 16th international conference on thermoelectrics (Cat. No. 97TH8291); 1997. P. 575-7.

9. Starner T. Human-powered wearable computing. IBM Syst J. 1996;35(3-4):618-29.

10. Altenkirch E. Uber den Nortzeffkt der Thermosaule. Phys. Z. 1909;10:560.

11. Ryan MA, Fleur JP. Where there is heat, there is a way. Electrochem. Soc. Interface. 2002;11(2):30-3.

12. Mansour A, Mohamed Hedi C, Faouzi B. Experimental study of a pack of supercapacitors used in electric vehicles. Sci World J. 2017. https://doi.org/10.1155/2017/6702838.

13. Zandi M, Payman A, Martin JP, Pierfederici S, Davat B, Meibody-Tabar F. Energy management of a fuel cell/ supercapacitor/battery power source for electric vehicular applications. IEEE Trans Veh Technol. 2011;60(2):433-43.

14. Gilbert JM, Balouchi F. Comparison of energy harvesting systems for wireless sensor networks. Int J Autom Comput. 2008;5(4):334-47.

15. Penella MT, Albesa J, Gasulla M. Powering wireless sensor nodes: primary batteries versus energy harvesting. In: 2009 IEEE instrumentation and measurement technology conference; 2009. P. 1625-30.

16. Sudevalayam S, Kulkarni P. Energy harvesting sensor nodes: survey and implications. IEEE Commun Surv Tutor. 2011;13(3):443-61.

17. Weddell AS, Merrett GV, Kazmierski TJ, Al-Hashimi BM. Accurate super capacitor modeling for energy harvesting wireless sensor nodes. IEEE Trans Circ Syst II: Express Briefs. 2011;58(12):911-5.

18. Zhang X, Zhang Z, Pan H, Salman W, Yuan Y, Liu Y. A portable high-efficiency electromagnetic energy harvesting system using supercapacitors for renewable energy applications in railroads. Energy Convers Manag. 2016;118:287-94. 